\title{
Posture and isokinetic shoulder strength in female water polo players
}

\author{
K D Aginsky, PhD; C Tracey, BHSc Hons Biokinetics; \\ N Neophytou, MSc (Med)
}

Centre for Exercise Science and Sports Medicine, School of Therapeutic Sciences, Faculty of Health Sciences, University of the Witwatersrand, Johannesburg, South Africa

\section{Corresponding author: K D Aginsky (kerithaginsky@gmail.com)}

Background: Being overhead athletes, water polo players can present with muscular imbalances of the shoulder, between the internal rotators (IR) and external rotators (ER), leading to changes in posture and an increased risk of injury.

Objectives: To assess posture and isokinetic shoulder strength of female club-level water polo players.

Methods: A descriptive study assessing posture and isokinetic strength of the IR and ER shoulder muscles in 15 female clublevel South African water polo players (age: $21.3 \pm 1.5$ years) was conducted. Posture was assessed using a posture grid. Isokinetic shoulder rotator muscle strength was tested over five repetitions concentrically and eccentrically at $60 \% \mathrm{sec}$ using a Biodex system 3 isokinetic dynamometer. The bilateral, reciprocal and functional dynamic control ratios (DCR) were calculated.

Results: Typical postures noted were a forward head, rounded shoulders, increased thoracic spine kyphosis, elevated non-dominant shoulder and mild scapula winging. The mean concentric reciprocal ratios for the dominant (52.2 \pm $7 \%)$ and non-dominant $(51.9 \pm 6.4 \%)$ sides indicated ER muscle weakness. DCR values were within normal limits for the group. (D: $0.75 \pm 0.2$ and ND: $0.75 \pm 0.1$ ).

Conclusion: There is a trend for these female water polo players to have rounded shoulders and forward head postures, as well as ER muscle strength weakness, the combination of which could predispose the athletes to shoulder injury.

Keywords: dynamic control ratio, shoulder injury, rounded shoulders, reciprocal ratio

S Afr J Sports Med 2016;28(3):64-68. DOI: 10.17159/2078-516X/2016/v28i3a438

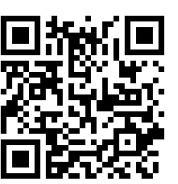

Water polo is a high-intensity intermittent aquatic sport which places large physical demands on the participants ${ }^{[1]}$ and involves repetitive physical motions that are common to swimming and baseball pitching. ${ }^{[2]}$ Water polo consists of four quarters, where two teams of seven players per side attempt to score goals by throwing the ball into their opponents' goal. Water polo is played in a pool measuring either $20 \mathrm{~m}$ by $10 \mathrm{~m}$ or $30 \mathrm{~m}$ by $20 \mathrm{~m}$, with a depth of $1.8 \mathrm{~m}$. During the throwing action, the mechanics are similar to those of baseball pitching. ${ }^{[2]}$ This forms a large component of the sport and involves the combination of muscle strength and coordination between the upper- and lower extremities. However, as the sport is played in a pool, unlike baseball or cricket, there are no stabilising surfaces ${ }^{[3]}$ from which to throw the ball. This, in turn, may increase the total forces on the joints at the shoulder complex.[2]

Water polo players continuously place their shoulder joints under recurring stress while performing repetitive overhead movements which can cause joint instability and muscular imbalances between the internal rotator (IR) and external rotator (ER) muscles. [2,4] Radaelli et al. [5] claims that this imbalance may occur due to the more frequent contraction pattern of the IR muscles compared to the ER muscles. The cumulative loads placed on the posterior shoulder joint during deceleration during throwing in water polo can result in posterior stiffness associated with a greater imbalance between the internal and external rotator cuff muscles and translation of the humeral head, thus predisposing the athlete to shoulder injury. [2]

Furthermore, during freestyle swimming in water polo, the head is more often out of the water and the ball positioned directly in front of the athlete[2,6] which places a heavy load on the shoulder joint and surrounding soft tissue.[7] In addition, while swimming freestyle in water polo, the dominant stroke action is that of head-up. This requires an altered form of traditional freestyle with the head out of the water and the arm elevation more exaggerated, as the arms are keeping the ball in front of the face of the player. Furthermore, to increase the force of the throw when shooting, a greater amount of external rotation and abduction are required, [6,8,9] enabling females for example to reach a speed to $16.8 \mathrm{~m} / \mathrm{s}$. [10] The amount of strength required to perform these forceful movements predispose the shoulder region to instability and muscle imbalance between the internal and external shoulder rotators. [11] Lynch et al. [12] reported that swimmers and overhead athletes often develop swimmer's shoulder which encompasses a variety of pathological injuries, such as rotator cuff tendinitis, shoulder instability and shoulder impingement.

A disparity in muscle balance may lead these athletes and swimmers to develop an increased risk of postural abnormalities and subsequently, to predispose them to shoulder injuries. However, there is limited evidence regarding the presence of muscle imbalances and postural abnormalities in water polo players. It has been established that if a malalignment in the posture is present, it may indicate that there is a muscle imbalance; thus postural orientation may play a role in sport performance. ${ }^{[13]}$ A study by Gradidge et al. showed that there is a relationship between poor shoulder posture and shoulder injury in water polo players. ${ }^{[14]}$

The limited research into posture and shoulder muscle strength in water polo players makes this study important in assisting with the identification of these muscle imbalances and specific postural characteristics, which will be useful in future studies. 


\section{Methods}

This was a descriptive study assessing the posture profiles and concentric and eccentric IR and ER shoulder muscle strength in 15 club-level female water polo players, aged between 18 and 25 years. Dominance was assessed as the preferred throwing arm. Ethical clearance was obtained from the Human Research Ethics Committee of the University of the Witwatersrand and written informed consent from each participant prior to testing. All participants were informed of the risk of muscle soreness, which is normal during the maximal isokinetic testing, prior to signing the informed consent. The study excluded any prospective participants with a shoulder, neck or back injury at the time of the testing, those who had suffered from a shoulder injury in the preceding six months, and those who had previous shoulder surgery, which would affect muscle strength output.

\section{Posture assessment}

The participants were asked to wear appropriate clothing so that the natural curves of the body could be seen. The subjects were asked to stand in their normal, comfortable anatomical position with the postural grid behind them and not to correct any postural abnormalities. A plumb line was used as a reference point in assessing the participant's posture. Each participant was analysed by an experienced therapist from an anterior, posterior and lateral view using the posture grid. Anterior and posterior views included the assessment of shoulder height, scoliosis, scapula winging and lateral pelvic tilting. The lateral assessment included head position, shoulder orientation, lumbar and thoracic curvatures, and anterior or posterior pelvic tilting. The various components were rated by one experienced researcher on the following scale: 0 = no abnormality, $1=$ slight, 2 = moderate, $3=$ severe .

\section{Isokinetic assessment}

Isokinetic strength was assessed using a Biodex system 3 isokinetic dynamometer (Biodex Medical Systems, Shirley, New York). Maximal strength testing of the IR and ER shoulder muscles was performed concentrically and eccentrically in the modified neutral position. Prior to testing, participants were warmed up on an arm ergometer (Technogym, Cesena, Italy) for five minutes. Participants were seated, with their upper body stabilised by means of stabilisation straps to prevent unwanted movement. The axis of rotation was aligned as the line from the olecranon process through the humerus to the acromion process, ensuring that the subject had full, safe range of motion. The participant was shown what will be required from them in the testing procedure. Testing included a standard Biodex strength testing protocol of five maximal concentric repetitions at $60 \% \mathrm{sec}$, followed by five maximal eccentric repetitions at $60 \%$ sec. The testing was performed on both of the subject's arms.

The following muscle strength ratios were calculated using the internal and external rotator muscle peak torque (PT) values:

- Reciprocal ratio (\%): (External rotator PT / Internal rotator PT) x 100
- Dynamic control ratio (Nm): Eccentric External rotator PT / Concentric Internal rotator PT

- $\quad$ Bilateral deficit (\%): (Dominant PT - Non-dominant PT) / Dominant PT x 100

- $\quad \mathrm{PT}$ to body weight $(\mathrm{Nm} / \mathrm{kg})$ : PT / body weight

\section{Statistical Analysis}

All data were descriptively analysed and are represented as means and standard deviations. A student's t-test was used to assess bilateral differences in the strength results. Significance was accepted at $\mathrm{p}<0.05$.

\section{Results}

\section{Demographic results}

Fifteen female club-level water polo players with a mean age of $21.3 \pm 1.5$ years were tested. They were $1.65 \pm 0.60 \mathrm{~m}$ tall and weighed $67.1 \pm 8.2 \mathrm{~kg}$. The majority of the participants were right-side dominant $(\mathrm{n}=14)$.

\section{Posture}

Figure 1 shows the head and shoulder orientation, characterised as slight, moderate or severe. Most of the athletes displayed a slight forward head posture $(n=11)$, whilst all had either a slight or moderate forward or rounded shoulder posture. Fourteen athletes had shoulder height discrepancies, with the majority having a slightly elevated non-dominant shoulder $(n=9)$. Furthermore, six players had slight scapula winging and one had moderate scapula winging. Nine players were observed to have slight thoracic kyphosis and four athletes displayed slight scoliosis.

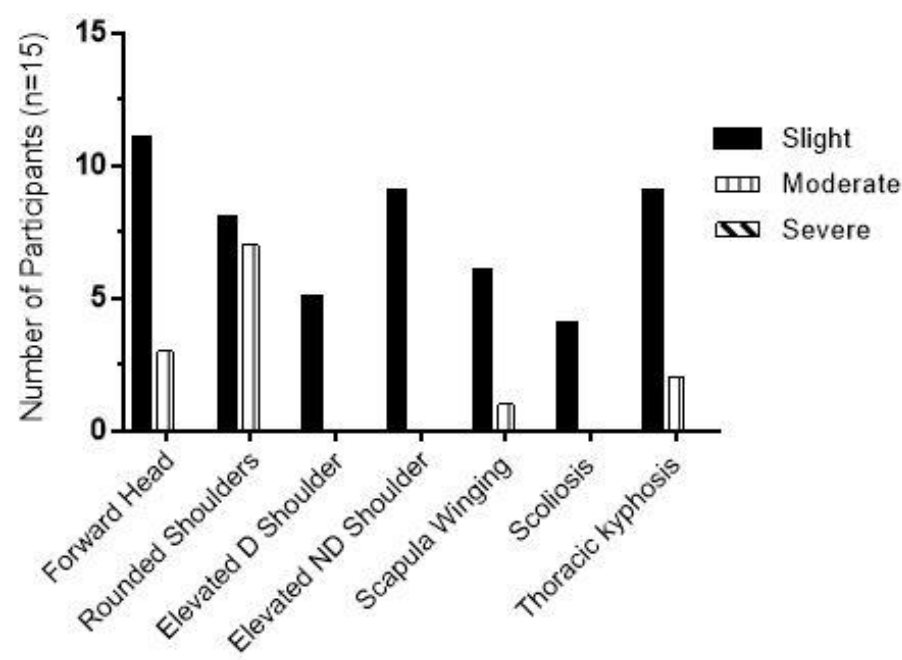

Fig 1. Upper body postural profile of 15 club-level female water polo players. D: Dominant; ND: Non-dominant

\section{Isokinetics}

\section{Peak torque (PT)}

Table 1 below shows the bilateral PT results for both concentric and eccentric tests for the water polo players. The ER peak torque values were lower than the IR peak torque values for both concentric and eccentric tests. There were no statistically significant bilateral differences found for either concentric $(p=$ 1.103; $\mathrm{p}=0.081)$ or eccentric peak torque values $(\mathrm{p}=1.199 ; \mathrm{p}=$ 0.207 ) for the internal and external shoulder rotators respectively. 
Table 1. Concentric and eccentric peak torque external and internal shoulder rotation values for female, club-level water polo players at $60 \%$ of dominant and non-dominant sides $(\mathrm{N}=15)$

\begin{tabular}{ccccc}
\hline Muscles & \multicolumn{2}{c}{ Dominant PT (N=15) } & \multicolumn{2}{c}{ Non-dominant PT (N=15) } \\
& Mean \pm SD & Range & Mean \pm SD & Range \\
\hline CON IR $(\mathrm{Nm})$ & $33.0 \pm 6.9$ & $22.5-46.1$ & $29.6 \pm 5.7$ & $20.4-40.3$ \\
CON ER (Nm) & $17.1 \pm 3.5$ & $12.0-23.7$ & $15.2 \pm 3.0$ & $10.6-21.2$ \\
ECC IR (Nm) & $34.9 \pm 5.7$ & $24.9-44.3$ & $32.7 \pm 6.1$ & $24.6-43.4$ \\
ECC ER $(\mathrm{Nm})$ & $24.0 \pm 5.1$ & $16.1-32.7$ & $21.9 \pm 4.6$ & 0.081 \\
\hline
\end{tabular}

PT: peak torque; CON: concentric; ECC: eccentric, IR: Internal rotators, ER: External rotators, Min: minimum, Max: maximum

Table 2. Dominant and non-dominant mean concentric and eccentric peak torque to body weight ratios at $60 \% \mathrm{~s}$ in female, club-level water polo players $(\mathrm{N}=15)$

\begin{tabular}{|c|c|c|c|c|c|}
\hline \multirow[t]{2}{*}{ Muscles } & \multicolumn{2}{|c|}{$\begin{array}{l}\text { Dominant PT/BW } \\
\qquad(\mathrm{N}=15)\end{array}$} & \multicolumn{2}{|c|}{$\begin{array}{l}\text { Non-dominant PT/BW } \\
(\mathrm{N}=15)\end{array}$} & \multirow[t]{2}{*}{$\mathbf{p}$} \\
\hline & Mean \pm SD & Range & Mean \pm SD & Range & \\
\hline CON IR (Nm/kg) & $0.49 \pm 0.1$ & $0.32-0.67$ & $0.44 \pm 0.1$ & $0.29-0.58$ & 0.143 \\
\hline CON ER $(\mathrm{Nm} / \mathrm{kg})$ & $0.25 \pm 0.0$ & $0.18-0.33$ & $0.22 \pm 0.0$ & $0.16-0.32$ & 0.136 \\
\hline ECC IR (Nm/kg) & $0.52 \pm 0.1$ & $0.39-0.67$ & $0.49 \pm 0.1$ & $0.38-0.59$ & 0.311 \\
\hline ECC ER $(\mathrm{Nm} / \mathrm{kg})$ & $0.36 \pm 0.1$ & $0.23-0.59$ & $0.32 \pm 0.1$ & $0.22-0.41$ & 0.244 \\
\hline
\end{tabular}

PT: peak torque; CON: concentric; ECC: eccentric, IR: Internal rotators, ER: External rotators, Min: minimum, Max: maximum

Table 3. Dominant and non-dominant mean concentric and eccentric reciprocal ratios at $60 \% \mathrm{~s}$ in female, club-level water polo players $(\mathrm{N}=15)$

\begin{tabular}{|c|c|c|c|c|c|}
\hline Ratio & Dominant $(\mathrm{N}=15)$ & Range (\%) & Non-dominant $(\mathrm{N}=15)$ & Range (\%) & $\mathbf{p}$ \\
\hline CON/CON (\%) & $52.2 \pm 7.0$ & 40 to 65 & $51.9 \pm 6.4$ & 40 to 62 & 0.914 \\
\hline $\mathrm{ECC} / \mathrm{ECC}(\%)$ & $69.9 \pm 16.0$ & 51 to 107 & $67.3 \pm 10.9$ & 55 to 93 & 0.652 \\
\hline
\end{tabular}

Con/Con; Concentric/ Concentric, Ecc/Ecc: Eccentric/ Eccentric

Table 4. Concentric and eccentric mean bilateral ratios for internal and external shoulder rotation at $60 \%$ in female club-level water polo players $(\mathrm{N}=15)$

\begin{tabular}{lllll}
\hline Contraction & IR (N=15) & Range (\%) & ER (N=15) & Range (\%) \\
\hline CON (\%) & $9 \pm 11$ & -6 to 33 & $10 \pm 6$ & -3 to 21 \\
ECC $(\%)$ & $6 \pm 10$ & -14 to 28 & $5 \pm 30$ & -80 to 49 \\
\hline CON; Concentric/ Concentric, ECC: Eccentric/ Eccentric, IR: Internal rotators, ER: External rotators
\end{tabular}

Table 5. Dominant and non-dominant mean dynamic control ratios at $60 \% \mathrm{~s}$ in female club-level water polo players (N=15)

\begin{tabular}{llllll}
\hline Ratio & Dominant $\mathbf{( N = 1 5 )}$ & Range & Non-dominant $\mathbf{( N = 1 5 )}$ & Range & $\mathbf{p}$ \\
\hline DCR & $0.75 \pm 0.21$ & 0.45 to 1.32 & $0.75 \pm 0.12$ & 0.57 to 0.92 & 0.984 \\
\hline DCR: Dynamic Control Ratio & & & &
\end{tabular}

\section{Peak torque/body weight ratio (PT/BW)}

$\mathrm{PT} / \mathrm{BW}(\mathrm{Nm} / \mathrm{kg})$ values were recorded for both concentric and eccentric contractions (Table 2). There were no bilateral differences for the concentric IR PT/BW $(\mathrm{p}=0.143)$ and concentric ER PT/BW $(p=0.136)$ respectively. The PT/BW ratios on the dominant and non-dominant sides were also similar when the eccentric IR $(\mathrm{p}=0.311)$ and ER PT/BW ratios $(p=0.244)$ were assessed.

\section{Reciprocal ratio}

The mean reciprocal ratios were calculated for both concentric and eccentric internal and external shoulder rotation on the dominant and non-dominant sides (Table 3). There were no bilateral differences for either the concentric $(p=0.914)$ or eccentric reciprocal ratios $(p=0.652)$. Although the mean values only show a slight ER muscle weakness, the range indicates that some athletes had a more pronounced ER muscle weakness for both concentric (40 to $62 \%$ ) and eccentric ratios
( $\mathrm{n}=4: 55$ to $60 \%$ ). For the non-dominant side $\mathrm{n}=11$ had a concentric ratio below $62 \%$ and $n=6$ for an eccentric ratio below $62 \%$.

\section{Bilateral deficit}

The bilateral ratio was calculated for concentric and eccentric internal and external shoulder rotation muscles (Table 4). The mean ratios were within normal limits for both the concentric and eccentric movement patterns and for both IR and ER muscle groups. However, when assessing the range there were athletes (Con ER: $n=8$ and Con IR: $n=6$; Ecc ER: $n=9$ and Ecc IR: $n=5$ ) who showed bilateral imbalances.

\section{Dynamic control ratio (DCR)}

The DCR for the dominant and non-dominant sides are shown in Table 5. There were no bilateral differences between the dominant and non-dominant arms $(\mathrm{p}=0.984)$. The range, however, indicates a high variation between the results of the 
participants (0.57 to 0.92$)$.

\section{Discussion}

The sport of water polo involves short bouts of high-intensity play with repetitive cyclic arm motions. [2] The assessment of water polo players' shoulder strength can assist in determining whether they have sufficient muscle strength to perform these tasks or whether there is a possible predisposition to injury.[15]

The demographic characteristics seen in the 15 female clublevel water polo players are comparable to other research on elite water polo players of a similar age. ${ }^{[16]}$ The majority of the participants presented with a forward head and rounded shoulder posture while nine also had slight thoracic spine kyphosis. These are common postures in swimmers and overhead athletes where they present with shortened cervical extensors and lengthened cervical flexors. $[3]$ Furthermore, the rounded shoulder posture may also indicate the presence of muscular imbalances surrounding the shoulder girdle, with the anterior chest muscles, such as the pectoralis major and minor being shortened. The posterior thoracic muscles, namely, the middle and lower trapezius and rhomboid muscles, were shown to be weak and lengthened. An imbalanced upper extremity posture would negatively affect the position of the glenohumeral joint and, combined with possible thoracic muscle weakness and fatigue, may predispose an individual to injury.[3, 12$]$

The majority of the athletes presented with an elevated nondominant $(n=9)$ or dominant $(n=5)$ shoulder. The presence of shoulder height discrepancy may be the result of a superiorly translated humeral head due to the lack of scapula stabilisation $[17]$ as seen by the thoracic kyphosis, rounded shoulder posture and winged scapulae. Superior translation of the humeral head can lead to a narrowing of the subacromial space and predisposition to rotator cuff impingement, which is due to postural imbalances seen in overhead athletes from weak external rotators compared to internal rotators. $\frac{[18]}{}$

The water polo players had lower peak torque values for the concentric and eccentric ER muscles compared to the IR muscles. Similarly, when peak torque was normalised to body weight, the IR muscle strength was greater than the ER muscle strength. These findings are further indicated by the weakness seen in the ER muscles relative to the IR muscles in the reciprocal ratio. These results are lower than previously found in the assessment of the peak torque to body weight ratio in asymptomatic overhead athletes ${ }^{[19]}$ and reciprocal ratio in water polo players. However, they were previously assessed at $30 \% / \mathrm{sec}$, which could account for the differences. ${ }^{[2]}$

It is important to also assess the comparison of the eccentric ER muscle strength relative to the concentric IR muscle strength in overhead athletes whose sport involves throwing. The concentric muscular contraction is important for the acceleration phase of throwing, whilst the eccentric muscle action is vital in the deceleration phase of throwing. [15] Thus the DCR evaluates the concurrent work of the muscles in terms of the strength of the eccentric ER strength relative to the concentric IR strength. Furthermore, this synchronisation of opposing muscles assists in the prevention of injury. The DCR for the group showed no bilateral differences (D: $0.75 \pm 0.21$ and ND: $0.75 \pm 0.12$ ); however, the large range on both sides indicates that there is a percentage of water polo athletes who show eccentric ER muscle weakness (D: 0.45 to 1.32 and ND: 0.57 to 0.92 ). These results demonstrate lower values than those found in previous studies, which were performed on non-water polo players. ${ }^{118]}$

The mean bilateral ratios for the group were within normal limits for both the concentric and eccentric movement patterns of the IR and ER muscle groups; however, when assessing the range, there were athletes who exhibited bilateral imbalances (Con IR: -6 to $33 \%$; Con ER: -3 to $21 \%$; Ecc IR: -14 to $28 \%$ and Ecc ER: -80 to $49 \%)$. These results indicate that within the group tested there are athletes who are possibly predisposed to injury. Similar findings in previous research, also found the dominant arm was stronger than the non-dominant arm in water polo players. [2] In addition, it has been found that athletes involved in overhead sports have a larger dominant arm than the non-dominant arm. [18] It is possible that this can be attributed to water polo, which is an asymmetrical sport, using mainly the dominant arm for throwing and shooting for goal. These muscle imbalances may be associated with the shoulder elevation found in the majority of water polo players; however, this relationship needs to be further investigated.

Thus the combination of postural abnormalities of the upper extremity and muscle weakness of the external shoulder rotators could predispose these athletes for developing shoulder injuries.

\section{Conclusion}

Female water polo players present with postural abnormalities, which include rounded and elevated shoulders, thoracic spine kyphosis and a forward head posture. These abnormalities are possibly associated with the concentric and eccentric weakness found in the external rotator muscles relative to the concentric internal rotator muscles. These muscle imbalances and postural abnormalities could predispose the water polo players to shoulder injuries.

Acknowledgements: The authors would like to thank the participants of the study.

Conflicts of interest: There are no conflicts of interest related to this study.

\section{References}

1. Mota N, Ribeiro F. Association between shoulder and proprioception and muscle strength in water polo players. Isokinet Exerc Sci 2012;20(1):17-21. doi: 10.3233/IES-2011-0435.

2. McMaster WC, Long SC, Caiozzo VJ. Isokinetic torque imbalances in the rotator cuff of the elite water polo player. Am J Sports Med 1991;19(1):72-75. doi: 10.1177/036354659101900112

3. Houglum P. Therapeutic exercise for musculoskeletal injuries. $3^{\text {rd }}$ ed 2010. Champaign, IL: Human Kinetics

4. Smith HK. Applied physiology of water polo. Sports Med 1998;26(5):317-334. doi: 10.2165/00007256-199826050-00003.

5. Radaelli R, Bottaro M, Weber F, et al. Influence of body position on shoulder rotator muscle strength during isokinetic assessment. Isokinet Exerc Sci 2010;18(3):119-124. doi: 10.3233/IES-2010-0369

6. Webster MJ, Morris ME, Galna B. Shoulder pain in water polo: a systematic review of the literature. J Sci Med Sport 2009;12(1):3-11. doi: 10.1016/j.jsams.2007.05.014

7. Elliott J. Shoulder pain and flexibility in elite water polo players. 
Physiotherapy1993;79(10):693-697.doi: http://dx.doi.org/10.1016/S00319406(10)60004-1

8. Giombini A, Rossi F, Pettrone FA, et al. Posterosuperior glenoid rim impingement as a cause of shoulder pain in top level waterpolo players. J Sport Med Phys Fitness 1997;37(4):273-278. doi: 10.1007/s00264-0101038-0

9. Witwer A, Sauers E. Clinical measures of shoulder mobility in college water-polo players. J Sports Rehabil 2006;15(1):45-57. doi: http://dx.doi.org/10.1123/jsr.15.1.45

10. Vila H, Manchado C, Rodriguez N, et al. Anthropometric profile, vertical jump, and throwing velocity in elite female handball players by playing positions. J Strength Cond. 2012;26(8):2146-2155. doi: 10.1519/JSC.0b013e31823b0a46

11. Bloomfield J, Blanksby B, Ackland T, et al. The influence of strength training on overhead throwing velocity of elite water polo players. Aust J Sci Med Sport 1990;22(9):205-220.

12. Lynch SS, Thigpen CA, Mihalik JP, et al. The effects of an exercise intervention on forward head and rounded shoulder postures in elite swimmers. Br. J. Sports Med. 2010;44(5):376-381. doi: 10.1136/bjsm.2009.066837

13. Sweeting K, Mock M. Gait and posture - assessment in general practice. Aust. Fam. Physician 2007;36(6):398-401,404-405. PMID 17565395
14. Gradidge P, Neophyton N, Benjamin N, et al. The injury and posture profiles of male high school water polo players in Johannesburg, South Africa. Afr. J. Phys. Health Educ. Recr. Dance. 2014;20(1):179-188

15. Ellenbecker TS, Davies GJ. The application of isokinetics in testing and rehabilitation of the shoulder complex. J. Athl. Train. 2000;35(3):338350. doi: 10.1016/B978-1-4377-2411-0.00025-3

16. Alcaraz PE, Abraldes JA, Ferragut C, et al. Throwing velocities, anthropometric characteristics, and efficacy indices of women's European water polo subchampions. J Strength Cond. 2011;25(11):30513058. doi: 10.1519/JSC.0b013e318212e20

17. Tovin BJ. Prevention and treatment of swimmer's shoulder. N Am J Sports Phys Ther 2006;1(4):166-175. PMCID: PMC2953356

18. van Cingel $R$, Kleinrensink GJ, Mulder $P$, et al. Isokinetic strength values, conventional ratio and dynamic control ratio of shoulder rotator muscles in elite badminton players. . Isokinet Exerc Sci 2007;15(4):287-293. 09593020

19. Zanca GG, Oliveira AB, Saccol MF, et al. Functional torque ratios and torque curve analysis of shoulder rotations in overhead athletes with and without impingement symptoms. J. Sports Sci. 2011;29(15):16031611. doi: 10.1080/02640414.2011.608702. 